\title{
SECOND LANGUAGE COMPOSITION TEACHING AT THE INTERMEDIATE LEVEL
}

\author{
Edward T. Erazmus \\ University of Michigan
}

With the emphasis among linguists in second language teaching on the oral-aural approach, the attention given to composition work has been negligible. The neglect is understandable for first things come first but it is to be recalled that Fries has said, "In the first stage of learning a new language, the end is that the basic structural patterns, with a limited vocabulary, are to be learned so well that they can be produced orally, automatically, and without hesitation, when the learner is confronted with the appropriate situation. To the accomplishment of this end, not only oral practice is used but also every other means of learning, including writing and reading." 1 Then follows the admonition, "After the first stage of language learning in accord with this approach the teacher or the student may devote himself entirely to reading and writing." 2 From these remarks it can be gathered that composition together with reading can play a relevant role in the second language learning process. It is also clear that an initial oral stage should be well under way if not completed before formal composition work begins. ${ }^{3}$

The term composition in this discussion will be taken to mean "free composition," that is, an original discourse created by the student about some given subject matter. Further, this discussion will assume that the learners are adults of the freshman college level type, or, in other words, the typical for eign student who has come to the States to receive or extend his college education: With a good oral course completed or well in progress-and this involves a basic vocabulary and all of the basic structures-the student is ready for formal composition work.

1Charles C. Fries, "American Linguistics and the Teaching of English," Language Leaming, VI (1955), p. 10.

2 Fries, p. 10.

$3_{\mathrm{A}}$ similar point of view appears in a policy statement, "On Foreign Language Teaching," in PMLA, LXXIV (Sept. 1959), vi . 
The linguistic condition of the intermediate student (one who has completed an oral course) indicates that he has a working familiarity with the basic vocabulary and the basic structures of the language. If his learning takes place largely under academic conditions, his control of the language will naturally be somewhat passive, artificial, and limited. The object of an intensive course, for example, is to get the student through the basic patterns of the language with the greatest speed possible. Likewise, since academic time is extremely limited, the patterns are practiced under strictly controlled stimulus conditions, resulting in a good but narrow control of the language even though all the work is done orally. There simply isn't time to deviate very much from the model sentences and patterns being taught. The learning under these conditions can be very rapid and extensive with regard to the number of patterns learned but the lack of flexibility and depth in pattern usage is evident. Written free composition can function as an effort in the academic situation to reinforce the work of the oral method and to extend the language control of the student. It is one of the best forms of closely observed language production. The student must reuse the patterns he has learned for his own communication purposes and this under complete observation of the teacher, for his written work is a faithful record of his usage.

When a student has reached the intermediate stage in learning a second language, there are four major problems confronting him in his attempts at composition. The first and most important of these is his limited ability with the language he is learning. There is no way to overcome this lack except by continued intensive work. The next large problem is interference in the use of the language from his own native language patterns. This is a well-known and well-described phenomenon. The interference decreases in proportion to the amount of exposure to the second language. The third large problem--and this has a direct effect on his composition style-is the interference of the stylistic and cultural literary expression patterns of his native language. Another difficulty, and this one is not linguistic, is that the student may have had little exposure to free composition in his native culture, making it a relatively unique experience. These difficulties conspire to make the first spontaneous composition efforts of the student appear childish, awkward, and full of grammatical errors. If by chance the student has a good control of the language, his efforts may have, depending on his background, the quality of being verbose, insincere, and filled with wordy, over-long sentences-features which are likely to be a reflex of his native manner of educated 
expression. If a rational view of the linguistic situation is taken, all of these limitations are completely predictable and should not offer the language teacher a reason for despair. The question which is most relevant is how to get these students to achieve an acceptable production of the written language in the most efficient and quickest manner.

When a student enters an intermediate program after completing a basic oral course there may be some hesitation on the part of the teachers to begin composition writing. The continuing lack in the oral production of the student may encourage a tendency to postpone free composition and concentrate on oral practice and the writing of various types of exercises which are intended to strengthen control of the oral patterns being taught. Some teachers may feel that the student's written work tends to be better than his oral work and on this account oral work should take precedence. While the oral method is without question the most efficient way of teaching a second language, it would be a disserivce to the student to postpone the benefits which can accure to him from free composition practice at this stage. It is also felt that proceeding through a program of graded writing involving such matters as paraphrasing, précis writing and other forms of imitative writing is a slow method of development and can be dispensed with in an intensive program. A suitable language program at the intermediate level would consist of continued controlled oral practice together with composition practice and work in reading.

To make free composition a useful instrument in the language development of the student, certain practical considerations have to be met. The first of these is that composition must be produced in great quantities to be effective. This follows the dictum of all language teaching that the establishment of language patterns as habits requires of the learner extensive practice in the features he is trying to learn. Facility with written forms of language and with patterns in general will be in direct proportion to the amount of usage they receive. This intensive production can be described in terms of the end product which it is intended to achieve, that is, fluency in written expression. By fluency in this context will be meant the ability of the student to produce a sustained discourse of some length on a subject familiar to him, in good informal prose, in a short period of time, with an adequate vocabulary and correct sentence patterns. For the foreign student to achieve this goal of fluency working under his linguistic handicaps, it is obvious that a reorientation from conventional composition teaching practice has to take place. The goals and forms of moti- 
vation which are presented to the student must be consistent with the linguistic capacity of the student. To speak of aesthetic values and style and such concepts as unity, logical development, and organization when the student is struggling to produce basic sentence patterns correctly with an adequate vocabulary is a loss of time. A different set of values for composition must prevail in this teaching situation.

Since the linguistic condition of the student is known and his limitations in the composition area can be predicted, the teacher should be prepared to receive poor copy in the initial stages. In language teaching we know, for example, that errors in pronunciation are due to the interference of the native phonemic system and are not a reflection of the student's intelligence, aptitude, and cooperation. This natural resistance to the new system is overcome by long hours of practice and the intelligent application of accumulated linguistic knowledge to the factors involved. Likewise, the poor effort of the student in his composition work is a reflex of his limited control of the language and cannot be interpreted as a lack of intelligence or capability. He enlarges his doman in written expression by extended use and practice under supervision. For this reason the student has to be pushed and motivated to produce extensively with little regard to the number and type of errors and infelicities he may make. These are corrected as they appear with the proper grammatical rationalization provided by the teacher. At a later stage the student should be able to diagnose his own errors. Under the program of fluency it is more important to have the student produce large quantities of material than to produce perfect copy. A high incidence of errors is not a cause for alarm. Errors and lapses are viewed as a consequence of the student's reaching into unfamiliar territory, using new complexes of vocabulary items and structures which he had probably never encountered before. It is quite natural that there should be awkward expressions, misused vocabulary terms and lapses in grammatical structure. But every new use of a vocabulary item, a variation in syntactical sequence, or a different structural combination is an enrichment of his language experience and an incorporation into his active language control of a new range of morphemes and their distribution. This is worth the errors that he makes. The next time he goes over similar territory he will do so with greater security and ease and with fewer mistakes. Continued extensive writing will do much toward establishing the written form of expression as an automatic habit. The same phenomenon is constantly happening in his oral use of language. However, written produc- 
tion makes greater demands upon the language potential of the user. This is considering the matter simply from the view of language involvement and does not include such matters as style, discourse content, and organization which are complexities on an entirely different level.

To achieve the goal of fluency certain precautions must be taken in the handling of student motivation. Fluency goals are not reached if students are spending long periods of time composing model compositions of 100 or 200 words in length. To demand perfection at this point is to put a limitation on language experience. Hence, the student is told frankly that a premium is placed on production, that his incidence of errors will be high and that he should write rapidly with little revision or recomposition, leaving the difficulties that he encounters in expression and syntax to the teacher. He is told further that it is much better to produce a 500 word composition than a 200 word composition and that a 1,000 word composition is much better than a 500 word one, etc. In the system of values that are projected, greatest emphasis is placed on length and the student is not devalued for the errors he makes. Nevertheless, the errors are pointed out very firmly and completely and he is told how he can overcome them, but he himself receives no discredit. As the students develop fluency, gradual pressure can be applied for more perfect copy. During classroom periods major student structural (and mechanical) errors will be discussed. Concurrent with such discussion should be a program of systematic grammar review as a part of the classroom activity. It is envisioned that eventually the teacher will be aided in his correction task by a codification of the major writing errors of the students. Such a codification, it is expected, will be based on a comparison of the structures of English and the native language of the student. Mechanics (spelling, punctuation, etc.) are secondary to language development and should not be used to bludgeon the student into submissiveness. Time permitting, mechanics can be treated briefly and systematically but the more technical aspects should be left for later English courses.

A further step in the promotion of fluency is the assignment and handling of subject content. The subject content of the composition is selected to fall within the personal experience of the student. Since an individual knows best his personal experiences and can describe them with the greatest ease, this forms the subject area that is most suitable to the fluency program. The foreign student, in that he is a representative of another culture, will have many interesting experiences in the 
area of culture contact. This provides a large source of subjects for discourse which is easily exploited. Further, the student is informed that he is a unique personality, different from any other personality in the world and that if he describes his experiences honestly, completely and directly without exaggeration and clichés, without trying to be "cute" or pedantic, his discourse will be of interest to his reader. This relieves the student of trying to live up to some misconceived literary standards and practices. Thus, a new set of values are offered to replace the complicated models of logical development, unity and coherence which are usually projected before the composition student. Interest and adequacy of treatment are the primary values in the area of content and organization. Inter est replaces the more formal demand for sophisticated subject matter, and adequacy of treatment and completeness of information replace logical and coherent development. In describing features of his culture or making cultural comparisons, the foreign student can be aided by providing him with certain elementary analytical and descriptive concepts which are familiar in linguistic analysis. Examples of such analytical procedures can be found in Chapter 6, "How To Compare Two Cultures," of Robert Lado's book, Linguistics Across Cultures. ${ }^{4}$ This involves the breaking down of any phenomenon into its features of meaning, distribution, and form, following the theory of Pike. ${ }^{5}$ Such an objective design will help the student provide maximum information about the subject he is treating, insuring that the description will be full and complete.

Another feature of the fluency program is the emphasis placed on rapid writing. In both his impromptus and in his homework compositions the student should be encouraged to write as rapidly as possible. As indicated above full information is the predominating criterion. With the de-emphasis on errors and stylistics, the student can write freely without the inhibitions often attending composition writing. He can even be told to limit the amount of time spent on his homework compositions so long as he produces adequately, thus freeing his time for other academic work. By handling large amounts of material in a short period of time with full information, the student will eventually sophisticate himself in the organization and handling of material. Under the program of fluency it fol-

\footnotetext{
4 (Ann Arbor, 1957).

5 Stated in Language in Relation to a Unified Theory of the Structure of Human Behavior, Part I, preliminary ed. (Glendale, Calif., 1954).
} 
lows that rewriting of compositions is of dubious value since it punishes the student who produces a great deal and rewards the student who writes briefly.

In the evaluation of the fluency program the total effect of the language process should be kept in mind. Language is a self-correcting and self-expanding system and the more that it is used the greater the facility there is in the use of it. At the primary level of instruction, that is, the oral level, the student is given, besides training in the sound system, intensive practice with basic grammatical structures and a limited vocabulary. He can become fluent within these limits but it is obvious that instruction cannot stop at this point. The objective is to increase fluency at increasingly higher levels of complexity until the student reaches the point where he can function with ease and skill at the social, economic, and academic level in which he intends to move. Hence, the aim at any level is to achieve fluency as rapidly as possible. This applies to written as well as to oral use of the language. The fact that this fluency is being controlled and observed by competent teachers will help to guarantee that non-native habits will be eliminated as they appear. To insist on perfection at any level is to inhibit fluency and to deny the student the benefits of expanding language experience.

In conclusion, it can be said that the primary end of the intermediate composition program is to strengthen and expand the student's productive control of language by the formal exercise of free composition. As in all language learning, the principle of extensive practice must apply for the student to achieve maximum control of the language patterns and of the medium of expression, that is, writing. To obtain maximum benefits, the student should be properly motivated and this means a reordering of the conventional teaching aims to suit the linguistic condition of the student and the linguistic goals that are sought. These goals are best expressed in the concept of fluency. In the practical area this means a restudy and re-evaluation of the elements that go into composition writing and projecting them so that they will offer the least resistance to maximum production. Under conditions of fluency the intermediate student will be doing the most for his present linguistic development and for his future academic work. 